University of Texas at El Paso

ScholarWorks@UTEP

\title{
How to Fuse Expert Knowledge: Not Always "And" but a Fuzzy Combination of "And" and "Or"
}

\author{
Christian Servin \\ El Paso Community College, cservin@gmail.com \\ Olga Kosheleva \\ The University of Texas at El Paso, olgak@utep.edu \\ Vladik Kreinovich \\ The University of Texas at El Paso, vladik@utep.edu
}

Follow this and additional works at: https://scholarworks.utep.edu/cs_techrep

Part of the Computer Sciences Commons

Comments:

Technical Report: UTEP-CS-19-09b

To appear in Proceedings of the World Congress of the International Fuzzy Systems Association and the Annual Conference of the North American Fuzzy Information Processing Society IFSA/ NAFIPS'2019,, Lafayette, Louisiana, June 18-22, 2019.

\section{Recommended Citation}

Servin, Christian; Kosheleva, Olga; and Kreinovich, Vladik, "How to Fuse Expert Knowledge: Not Always "And" but a Fuzzy Combination of "And" and "Or"'" (2019). Departmental Technical Reports (CS). 1285. https://scholarworks.utep.edu/cs_techrep/1285

This Article is brought to you for free and open access by the Computer Science at ScholarWorks@UTEP. It has been accepted for inclusion in Departmental Technical Reports (CS) by an authorized administrator of ScholarWorks@UTEP. For more information, please contact Iweber@utep.edu. 


\title{
How to Fuse Expert Knowledge: Not Always "And" but a Fuzzy Combination of "And" and "Or"
}

Christian Servin, Olga Kosheleva, and Vladik Kreinovich

\begin{abstract}
In the non-fuzzy (e.g., interval) case, if two expert's opinions are consistent, then, as the result of fusing the knowledge of these two experts, we take the intersection of the two sets (e.g., intervals) describing the expert's opinions. In the experts are inconsistent, i.e., if the intersection is empty, then a reasonable idea is to assume that at least of these experts is right, and thus, to take the union of the two corresponding sets. In practice, expert opinions are often imprecise; this imprecision can be naturally described in terms of fuzzy logic - a technique specifically designed to describe such imprecision. In the fuzzy case, expert opinions are not always absolutely consistent or absolutely inconsistent, they may be consistent to a certain degree. In this case, we show how the above natural idea of fusing expert opinions can be extended to the fuzzy case. As a result, we, in general, get not "and" (which would correspond to the intersection), not "or" (which would correspond to the union), but rather an appropriate fuzzy combination of "and"- and "or"-operations.
\end{abstract}

\section{Fusing Expert Knowledge: Formulation of the Problem}

Need to fuse knowledge of different experts. Expert estimates of different quantities are usually not very accurate - e.g., in situations when measurements are also possible, measurement results are usually much more accurate than expert estimates.

When we can perform measurements, we can further increase the measurement accuracy if we use several different measuring instruments and then combine

\section{Christian Servin}

Computer Science and Information Technology Systems Department

El Paso Community College, 919 Hunter, El Paso, TX 79915, USA, e-mail: cservin@ gmail.com

Olga Kosheleva, and Vladik Kreinovich

University of Texas at El Paso, El Paso, Texas 79968, USA

e-mail: olgak@utep.edu,vladik@utep.edu 
("fuse") their results. It is known that such combinations are usually more accurate than all original measurement results.

In many situations, measurements are not realistically possible, so we have to rely on expert estimates only. In such situations, we can increase the accuracy of the resulting estimates the same way we increase the accuracy of measurement results: by combining (fusing) estimates of several experts.

Examples. To estimate the temperature, we can ask two experts. Suppose that:

- one expert states that the temperature is between 22 and 25 degree $\mathrm{C}$, and

- another expert states the temperature is in the low seventies, i.e., between 70 and $75 \mathrm{~F}$ - which corresponds to between 21 and $24 \mathrm{C}$.

Then we can conclude that the actual temperature is larger than $22 \mathrm{C}$ and smaller than $24 \mathrm{C}$ - i.e., the actual temperature is between 22 and $24 \mathrm{C}$.

In this case, if we only asked one expert, we would have an interval of width 3 that contains the actual (unknown) temperature value. But by fusing the opinions of the two experts, we get a narrower interval $[22,24]$ of width 2 - i.e., we have indeed increased the accuracy.

Fusion is also possible on a non-quantitative level. For example, we can ask experts whether the wind is weak, moderate, or strong. Suppose that:

- one expert says that the wind is not weak, while

- another expert says that the wind is not strong.

By combining the opinions of both experts, we can conclude that the wind is moderate.

On the other hand, if we only to one of the experts, we would not be able to come to this conclusion:

- if we only took into account the opinion of the first expert, then we would only be able to conclude that the wind is either moderate or strong;

- similarly, if we only took into account the opinion of the first expert, then we would only be able to conclude that the wind is either moderate or weak.

Fusing expert knowledge: non-fuzzy case. To understand how to best combine expert estimates, let us start with the case when expert estimates are crisp (nonfuzzy), i.e., when for each possible value of the estimated quantity, the expert is either absolutely sure that this value is possible or is absolutely sure that the given value is not possible. In this case, each expert estimate provides us with a set of possible values of the corresponding quantity. In most practical cases, this set is an interval $[\underline{x}, \bar{x}]$.

In these terms, when we have estimates of two different experts, this means that:

- based on the opinions of the first expert, we form a set $S_{1}$ of numbers which are, according to this expert, possible values of the estimates quantity;

- also, based on the opinions of the second expert, we form a set $S_{2}$ of numbers which are, according to this expert, possible values of the estimates quantity. 
In general, different experts take into account different aspects of the situation. For example, the first expert may know the upper bound $\bar{x}$ on the corresponding quantity. In this case, the set $S_{1}$ consists of all the numbers which are smaller than or equal to $\bar{x}$, i.e., $S_{1}=(-\infty, \bar{x}]$. The second expert may know the lower bound $\underline{x}$, in which case $S_{2}=[\underline{x}, \infty)$. In such situations, a natural way to fuse the knowledge is to consider numbers which are possible according to both experts, i.e., in mathematical terms, to consider the intersection $S_{1} \cap S_{2}$ of the two sets $S_{1}$ and $S_{2}$.

A problem occurs when this intersection is empty, i.e., when the opinions of two experts are inconsistent. This happens: experts are human and can thus make mistakes. In this case, an extreme option is to say that since experts are not consistent with each other, this means that we do not trust what each of them says, so we can as well ignore both opinions; the result of fusion is then the whole real line.

A more reasonable option is to conclude that, yes, both experts cannot be true, but we cannot conclude that both are wrong; they are experts after all, so it is reasonable to assume that one of them is right; in this case, the result of the fusion is the union $S_{1} \cup S_{2}$ of the two sets.

In other words, the fusion $S_{1} f S_{2}$ of the sets $S_{1}$ and $S_{2}$ has the following form;

- if $S_{1} \cap S_{2} \neq \emptyset$, then $S_{1} f S_{2}=S_{1} \cap S_{2}$;

- otherwise, if $S_{1} \cap S_{2}=\emptyset$, then $S_{1} f S_{2}=S_{1} \cup S_{2}$.

Example. Suppose that:

- one expert says that the temperature is between 22 and 25 , and

- another one claims that it is between 18 and 21 .

In this case, the intersection of the corresponding intervals $[22,25]$ and $[18,21]$ is empty - which means that the experts cannot be both right. What we can conclude if we still believe that one of them is right - is that the temperature is either between 22 and 25 or between 18 and 21.

Need to consider the fuzzy case. In practice, experts are rarely absolutely confident about their opinions. Usually, they are only confident to a certain degree. As a result, to adequately describe expert knowledge, we need to describe, for each number $x$, the degree to which, according to this expert, the number $x$ is possible. This is the fuzzy logic approach; see, e.g., [1, 2, 3, 4, 5, 6, 7].

In the computer, "true" (= "absolute certain") is usually represented as 1 , and "false" (= "absolutely certain this is false") is represented as 0 . It is therefore reasonable to describe intermediate degrees of confidence by numbers intermediate between 0 and 1 . Thus, to describe an expert's estimate, we need to have a function $\mu(x)$ that assigns, to each value $x$ of the corresponding quantity, a number $\mu(x) \in[0,1]$ that describes to what extent the value $x$ is possible. Such a function is known as a membership function, or, alternatively, a fuzzy set. From this viewpoint, to be able to fuse expert estimates, we need to be able to fuse fuzzy sets.

A traditional approach to fusing fuzzy knowledge simply takes the intersection which is then usually normalized, i.e., multiplied by a constant so that the maximum value is 1 . However, this does not work if the expert opinions are inconsistent. We 
should therefore take into account that the expert opinions can be inconsistent - or, more generally, consistent to a certain degree. How can take this into account?

What we do in this paper. In this paper, we show how to extend the above fusion operation to the fuzzy case.

\section{Analysis of the Problem}

In general, how notions are generalized to the fuzzy case. The usual way to generalize different notions to the fuzzy case is as follows:

- First, we describe the original notion in logical terms, by using "and', "or", and quantifiers "for all" (which is, in effect, infinite "and") and "exists" (which is, in effect, infinite "or").

- Then, we replace each "and" operation with the fuzzy "and"-operation $f_{\&}(a, b)$ (also known as t-norm) and every "or"-operation with the fuzzy "or"-operation $f_{\vee}(a, b)$ (also known as t-conorm).

In selecting the t-norms and t-conorms, we need to be careful, in the following sense.

- If we have a universal quantifier - i.e., an infinite "and" - and we use, e.g., a product t-norm $f_{\&}(a, b)=a \cdot b$, then the product of infinitely many values smaller than 0 will be most probably simply 0 . So, if we have an infinite "and" (= universal quantifier), the only t-norm that leads to meaningful results is the minimum

$$
f_{\&}(a, b)=\min (a, b) .
$$

- Similarly, if we have an existential quantifier - i.e., an infinite "or" - and we use, e.g., an algebraic sum t-conorm $f_{\vee}(a, b)=a+b-a \cdot b$, then the result of applying this operation to infinitely many values larger than 0 will be most probably simply 1 . So, if we have an infinite "or" (= existential quantifier), the only $\mathrm{t}$-conorm that leads to meaningful results is the maximum

$$
f_{\vee}(a, b)=\max (a, b) .
$$

How to define degree of consistency. Let us use the above-described general approach to define the degree of consistency. In the non-fuzzy case, two expert opinions are consistent if there exists a value $x$ for which both the first expert and the second expert agree that this value is possible.

For each real number $x$ representing a possible value of the quantity of interest:

- let $\mu_{1}(x)$ denote the degree to which the first expert believes the value $x$ to be possible, and

- let $\mu_{2}(x)$ denote the degree to which the second experts believes the value $x$ to be possible. 
Then, for each value $x$, the degree to which both experts consider the value $x$ to be possible if equal to $f_{\&}\left(\mu_{1}(x), \mu_{2}(x)\right)$, where $f_{\&}(a, b)$ is an appropriate fuzzy "and"operation (t-norm).

In line with the above general scheme for generalizing notions into fuzzy, the existential quantifier over $x$ is translated into maximum over $x$ (which corresponds to the use of the maximum "or"-operation $f_{\vee}(a, b)=\max (a, b)$ ). Thus, we get the following formula for the degree $d\left(\mu_{1}, \mu_{2}\right)$ for which two membership functions are consistent:

$$
d\left(\mu_{1}, \mu_{2}\right)=\max _{x} f_{\&}\left(\mu_{1}(x), \mu_{2}(x)\right) .
$$

Accordingly, in line with a general description of negation in fuzzy logic, the degree to which the expert opinions are inconsistent can be computed as

$$
1-d\left(\mu_{1}, \mu_{2}\right)
$$

Resulting definition of fusion. According to the definition given in the previous section, in the non-fuzzy case, the value $x$ belongs to the fused set if:

- either the two sets describing expert opinions are consistent, and $x$ belongs to the intersection of the two sets,

- or the two sets describing expert opinions are inconsistent, and $x$ belongs to the union of these two sets.

Let us use the general methodology to generalize the above description to the fuzzy case. For each $x$ :

- we know the degree $d\left(\mu_{1}, \mu_{2}\right)$ to which the experts are consistent, and

- we know the degree $f_{\&}\left(\mu_{1}(x), \mu_{2}(x)\right)$ to which $x$ belongs to the intersection.

Thus, the degree to which the expert opinions are consistent and $x$ belongs to the intersection can be obtained by applying the "and"-operation $f_{\&}(a, b)$ to these two degrees. Thus, we get the value

$$
f_{\&}\left(d\left(\mu_{1}, \mu_{2}\right), f_{\&}\left(\mu_{1}(x), \mu_{2}(x)\right)\right)=f_{\&}\left(d\left(\mu_{1}, \mu_{2}\right), \mu_{1}(x), \mu_{2}(x)\right) .
$$

Similarly, for each $x$ :

- we know the degree $1-d\left(\mu_{1}, \mu_{2}\right)$ to which the experts are inconsistent, and

- we know the degree $f_{\vee}\left(\mu_{1}(x), \mu_{2}(x)\right)=\max \left(\mu_{1}(x), \mu_{2}(x)\right)$ to which $x$ belongs to the union.

Thus, the degree to which the expert opinions are inconsistent and $x$ belongs to the union can be obtained by applying the "and"-operation $f_{\&}(a, b)$ to these two degrees. Thus, we get the value

$$
f_{\&}\left(1-d\left(\mu_{1}, \mu_{2}\right), \max \left(\mu_{1}(x), \mu_{2}(x)\right)\right) .
$$


To find the degree $\mu(x)$ to which the value $x$ belongs to the fused set, we need to apply the "or"-operation $f_{\vee}(a, b)=\max (a, b)$ to the degrees (2) and (3). As a result, we get the following formula.

\section{Resulting Formula: Formulation and Example}

Resulting formula: general case. If we know the functions $\mu_{1}(x)$ and $\mu_{2}(x)$ that describe the opinions of the two experts, then, to describe the fused opinion, we should take the function

$$
\mu(x)=\max \left(d_{1}(x), d_{2}(x)\right)
$$

where

$$
\begin{gathered}
d_{1}(x) \stackrel{\text { def }}{=} f_{\&}\left(d\left(\mu_{1}, \mu_{2}\right), \mu_{1}(x), \mu_{2}(x)\right), \\
\left.d_{2}(x) \stackrel{\text { def }}{=} f_{\&}\left(1-d\left(\mu_{1}, \mu_{2}\right), \max \left(\mu_{1}(x), \mu_{2}(x)\right)\right)\right),
\end{gathered}
$$

$f_{\&}(a, b)$ is an "and"-operation (t-norm) and the degree $d\left(\mu_{1}, \mu_{2}\right)$ is determined by the formula

$$
d\left(\mu_{1}, \mu_{2}\right)=\max _{x} f_{\&}\left(\mu_{1}(x), \mu_{2}(x)\right) .
$$

Discussion. We can see that this fused fuzzy set is not exactly "and", it is not exactly "or" - it is a fuzzy combination of "and" and "or".

Case when $f_{\&}(a, b)=\min (a, b)$. In the case when, as the "and"-operation, we select the simplest possible "and"-operation $f_{\&}(a, b)=\min (a, b)$, the above formulas (4)(6) can be further simplified. Namely, by definition of the degree of consistency $d\left(\mu_{1}, \mu_{2}\right)$, this degree is the largest of the values $f_{\&}\left(\mu_{1}(x), \mu_{2}(x)\right)$. Thus, for every $x$, we have $f_{\&}\left(\mu_{1}(x), \mu_{2}(x)\right) \leq d\left(\mu_{1}, \mu_{2}\right)$. Therefore, since our "and"-operation is minimum, we get a simplified expression for the formula (2):

$$
f_{\&}\left(d\left(\mu_{1}, \mu_{2}\right), f_{\&}\left(\mu_{1}(x), \mu_{2}(x)\right)=f_{\&}\left(\mu_{1}(x), \mu_{2}(x)\right)=\min \left(\mu_{1}(x), \mu_{2}(x)\right) .\right.
$$

Thus, for the minimum "and"-operation, the formulas (4)-(6) take the following simplified form:

$$
\mu(x)=\max \left(\min \left(\mu_{1}(x), \mu_{2}(x)\right), \min \left(1-d\left(\mu_{1}, \mu_{2}\right), \max \left(\mu_{1}(x), \mu_{2}(x)\right)\right)\right) .
$$

Example. To illustrate the above formula, let us consider a simple case when the "and"-operation is minimum, and the membership functions are triangular functions of the same width. To make the computations even easier, let us select, as a starting point for measuring $x$, the arithmetic average between the most probable values 
corresponding to the two experts, and let us select the measuring unit so that the half-width of each membership function is 1 .

In this case, the triangular membership functions are described by the formulas $\mu_{1}(x)=\max (0,1-|x-a|)$ and $\mu_{2}(x)=\max (0,1-|x+a|)$, for some $a>0$. This value $a$ is the half of the difference between the most probable value (a) according to the first expert and the most probable value according to the second expert $(-a)$ : $a=\frac{a-(-a)}{2}$.

When $a \geq 1$, the two membership functions have no intersection at all, so $d\left(\mu_{1}, \mu_{2}\right)=0$, and the fused set is simply their union $\max \left(\mu_{1}(x), \mu_{2}(x)\right)$, a bi-modal set whose graph consists of the two original triangles.

The more interesting case is when $a<1$. In this case, the two sets have some degree of intersection. For such values $a$, as one can easily check, the intersection $f_{\&}\left(\mu_{1}(x), \mu_{2}(x)\right)$ is also a triangular function $\max (0,1-a-|x|)$. The maximum $d\left(\mu_{1}, \mu_{2}\right)$ of this function is attained when $x=0$ and is equal to $1-a$.

Correspondingly, the degree to which the two expert opinions are inconsistent is equal to $1-d\left(\mu_{1}, \mu_{2}\right)=1-(1-a)=a$. By applying the formula (7), we can now conclude the following.

When $a \leq 0.5$, the fused expression is still a fuzzy number, i.e., a membership function which first increases and then decreases. Specifically:

- The fused function $\mu(x)$ starts being non-zero at the value $x=-1-a$; between the value $-1-a$ and -1 , it grows as $\mu(x)=x-(-1-a)=1+a-x$.

- Between the values $x=-1$ and $x=-(1-2 a)$, the fused function remains constant $\mu(x)=a$.

- Between $x=-(1-2 a)$ and $x=0$, it grows as $\mu(x)=1-a+x$, until it reaches the value $1-a$.

- Then, for $x$ from 0 to $1-2 a$, it decreases as $\mu(x)=1-a-x$ until it reaches the value $a$ for $x=1-2 a$.

- Then, the value stays constant $\mu(x)=a$ until we reach $x=1$.

- Finally, for $x$ between 1 and $1+a$, the values decreases as $\mu(x)=(1+a)-x$, until it reaches 0 for $x=1+a$ - and stays 0 after that.

We can normalize the resulting function, by dividing it by its largest possible value $1-a$. Then, the constant levels increase to $\frac{a}{1-a}$.

When $a>0.5$, we simply get the union cut-off at level $1-a$, i.e.,

$$
\mu(x)=\min \left(1-a, \max \left(\mu_{1}(x), \mu_{2}(x)\right)\right) .
$$

\section{Acknowledgments}

This work was supported in part by the US National Science Foundation via grant HRD-1242122 (Cyber-ShARE Center of Excellence).

The authors are thankful to the anonymous referees for valuable suggestions. 


\section{References}

1. R. Belohlavek, J. W. Dauben, and G. J. Klir, Fuzzy Logic and Mathematics: A Historical Perspective, Oxford University Press, New York, 2017.

2. G. Klir and B. Yuan, Fuzzy Sets and Fuzzy Logic, Prentice Hall, Upper Saddle River, New Jersey, 1995.

3. J. M. Mendel, Uncertain Rule-Based Fuzzy Systems: Introduction and New Directions, Springer, Cham, Switzerland, 2017.

4. H. T. Nguyen and V. Kreinovich, "Nested intervals and sets: concepts, relations to fuzzy sets, and applications", In: R. B. Kearfott and V. Kreinovich (eds.), Applications of Interval Computations, Kluwer, Dordrecht, 1996, pp. 245-290.

5. H. T. Nguyen, C. Walker, and E. A. Walker, A First Course in Fuzzy Logic, Chapman and Hall/CRC, Boca Raton, Florida, 2019.

6. V. Novák, I. Perfilieva, and J. Močkoř, Mathematical Principles of Fuzzy Logic, Kluwer, Boston, Dordrecht, 1999.

7. L. A. Zadeh, "Fuzzy sets", Information and Control, 1965, Vol. 8, pp. 338-353. 